\title{
Conhecimento sobre mudanças climáticas implica em adaptação? Análise de agricultores do Nordeste brasileiro
}

\author{
Does knowledge about climate change imply \\ adaptation? Analysis of farmers in Northeast Brazil
}

\author{
Sabrina de Matos Carlos ${ }^{1}$ (D), Dênis Antônio da Cunha ${ }^{1}$
}

e Marcel Viana Pires ${ }^{1}$

Resumo: Este estudo analisa a relação entre conhecimento sobre as mudanças climáticas e adoção de estratégias adaptativas de agricultores do Nordeste (bacia hidrográfica do Rio das Contas, Bahia). Metodologicamente, foi estimado um modelo Probit Bivariado Aparentemente Não Relacionado. Os resultados evidenciaram que conhecimento e adaptação são correlacionados e devem ser pensados de forma conjunta. Agricultores que conhecem os efeitos das mudanças climáticas têm maior probabilidade de adotar medidas adaptativas. Isso reforça a importância de se considerar a percepção, o conhecimento e as condições socioeconômicas dos indivíduos na formulação de políticas públicas de combate aos efeitos das mudanças climáticas.

Palavras-chave: conhecimento, adaptação, mudanças climáticas, bacia hidrográfica do Rio das Contas, probit bivariado aparentemente não relacionado.

Abstract: We analyze the relationship between farmer's knowledge about climate change and the adoption of adaptive strategies. The study area was the Brazilian Northeast (Rio das Contas basin, Bahia). Methodologically, we estimated a Seemingly Unrelated Bivariate Probit model. The results showed that knowledge and adaptation are correlated and should be considered together. Farmers who are aware of the climate change effects are more likely to adapt. This finding reinforces the importance of considering the perception, knowledge and socioeconomic conditions of individuals in the formulation of public policies to counteract the negative effects of climate change.

Key-words: knowledge, adaptation, climate change, Rio das Contas basin, seemingly unrelated bivariate probit model.

Classificação JEL: C25, Q5, O13, R11.

Data de submissão: 8 de novembro de 2017. Data de aceite: 28 de julho de 2018.

1 Universidade Federal de Viçosa (UFV), Viçosa (MG), Brasil. E-mails: sabrinamcarlos@gmail.com; denis.cunha@ufv.br; marcelpires@gmail.com 


\section{Introdução}

As mudanças climáticas globais constituem-se o principal desafio a ser enfrentado por todas as sociedades no século XXI, já que seus impactos, em geral negativos, serão sentidos distintamente entre as diversas atividades econômicas e localidades ao redor do planeta. As pesquisas sobre o tema não permitem previsão precisa da extensão ou distribuição dos impactos, mas destacam efeitos negativos expressivos sobre a saúde humana, os ecossistemas e a biodiversidade, os recursos hídricos e, sobretudo, a produção agropecuária (Intergovernmental Panel on Climate Change, 2014).

Segundo o Quinto Relatório de Avaliação do Intergovernmental Panel on Climate Change (2014), a maior parte das pesquisas realizadas em termos dos possíveis impactos das mudanças climáticas sugere que os países pobres, sobretudo aqueles localizados em baixas latitudes, serão os mais prejudicados. Esses países estão situados em regiões onde as condições climáticas já são adversas, possuem baixa capacidade adaptativa e apresentam alta dependência em relação ao setor primário, responsável por expressiva parcela de seu Produto Interno Bruto (PIB).

No caso brasileiro, em que o agronegócio responde por cerca de 22\% do PIB (Centro de Estudos Avançados em Economia Aplicada, 2015), as mudanças climáticas podem causar fortes impactos negativos. Estudos que analisaram o Brasil indicam que o país terá perdas econômicas decorrentes das alterações climáticas, que podem variar desde valores pouco expressivos no curto prazo até grandes prejuízos no longo prazo (Nelson et al., 2014; Pires \& Cunha, 2014; Rosenzweig et al., 2014; Cunha et al., 2015; Schembergue et al., 2017). De acordo com Nelson et al. (2014), os impactos das mudanças climáticas poderiam causar redução média na produtividade das principais culturas produzidas no país de aproximadamente $22 \%$ até 2050 . Além disso, os preços médios dos produtos agrícolas aumentariam em 5\%. A concretização dessas expectativas poderia causare agravar diversos problemas socioeconômicos brasileiros, tais como pobreza rural e urbana, fluxos migratórios e insegurança alimentar.

Nesse sentido, estratégias de adaptação às mudanças climáticas são essenciais para que os agricultores e todos os elos da cadeia produtiva do agronegócio possam minorar os efeitos adversos previstos. Moore \& Lobell (2014) destacam que projetar os impactos das mudanças climáticas na agricultura exige primeiramente compreender como os produtores se adaptarão e quais são os principais direcionadores desse processo. Sendo assim, o conhecimento dos fatores que desencadeiam a resposta dos agricultores às alterações do clima é crucial para o efetivo desenvolvimento de políticas de enfrentamento ao problema (Blennow et al., 2012). É fundamental que as políticas públicas levem em consideração não só a necessidade/capacidade de adaptação, mas também a percepção e o conhecimento que os agricultores têm acerca das mudanças climáticas. O conhecimento do fenômeno por parte daqueles que serão alvo das políticas certamente será fator decisivo para o seu sucesso e eficiência. 
Nesse sentido, o presente trabalho procurou responder à uma questão principal: o conhecimento sobre mudanças climáticas por parte de agricultores implica em adaptação? Especificamente, a pesquisa teve como objetivos (i) compreender a percepção sobre mudanças climáticas dos agricultores; (ii) avaliar como o conhecimento/percepção influencia a decisão de adaptação dos agricultores e (iii) analisar as variáveis socioeconômicas mais importantes na execução das estratégias adaptativas.

O trabalho procurou dar enfoque local à questão de pesquisa ao estudar a região da bacia hidrográfica do Rio das Contas, Bahia. A escolha da área de estudo justifica-se pelo fato de a região Nordeste ser uma das que potencialmente serão mais afetadas pelas alterações climáticas futuras no Brasil. A literatura climática indica que nessa região ocorrerão os maiores aumentos de temperatura e grande variabilidade interanual da precipitação durante a estação chuvosa (dezembro-fevereiro). Os estudos também concluem que a produção e a aptidão agrícola nordestina serão comprometidas e que outros problemas já sentidos na região (como pobreza, insegurança alimentar, degradação da terra e da biodiversidade e competição por água e energia) serão agravados (Intergovernmental Panel on Climate Change, 2014; Machado-Filho, 2015; Nunes, 2016). Além disso, a região da bacia hidrográfica do Rio das Contas possui características que a torna possivelmente mais vulnerável aos efeitos adversos do clima. Por exemplo, é composta por cidades de pequeno a médio porte, com baixo Índice de Desenvolvimento Humano e atividade econômica centrada, principalmente, na agricultura e pecuária (Paula et al., 2010). Por essa razão, a adoção de estratégias adaptativas é essencial para minimizar os efeitos negativos previstos e evitar maior empobrecimento regional.

De modo geral, acredita-se que a presente pesquisa avança devido ao seu caráter regional e também por considerar explicitamente o papel do conhecimento / percepção acerca do fenômeno na adoção de estratégias adaptativas. No Brasil esses temas ainda não têm sido abordados, embora internacionalmente haja crescente literatura que busca compreender como o conhecimento afeta a adaptação. Os resultados encontrados e as discussões acerca do tema evidenciam que o conhecimento tem papel determinante sobre o delineamento das estratégias de adaptação. Segundo
Nguyen et al. (2016), a decisão dos agricultores quanto à adaptação tem como base o conhecimento e a forma como interpretam as informações climáticas disponíveis. Além disso, a adaptação às mudanças climáticas é um processo local que é arraigado às relações sociais estabelecidas entre os agricultores e ao aprendizado compartilhado. Portanto, não é possível implementar uma estratégia de adaptação sem considerar a forma pela qual o conhecimento é moldado (Kpadonou et al., 2012; Lebel, 2013; Naess, 2013). A integração entre o conhecimento e adaptação e seus possíveis benefícios têm sido pouco explorados durante o desenvolvimento das políticas públicas voltadas à problemática das mudanças climáticas.

A atenção crescente dada às mudanças climáticas não tem sido acompanhada por tentativas de compreender o papel do conhecimento sobre a adoção de medidas adaptativas, sobretudo em âmbito regional. No Brasil são raras as análises que tratam do tema. Dessa forma, pretende-se dar suporte à formulação de políticas públicas direcionadas à região e aos atores nela envolvidos, além de auxiliar nas medidas futuras que visem a melhor compreensão dos direcionadores dos processos adaptativos. A existência de estudos regionais sobre mudanças climáticas no âmbito da agricultura brasileira é reforçada tendo por base as distintas realidades existentes nas mais diversas regiões do país. Para Andrade et al. (2015), os resultados de programas ou políticas públicas universais ${ }^{1}$ voltados para agricultura nacional estão diretamente ligados às características locais. Sendo assim, o fortalecimento e a busca de melhores resultados para essas políticas a nível local podem ser obtidos por meio do conhecimento prévio das questões sociais, culturais, ambientais, políticas e institucionais que estão envoltas no setor agrícola regional.

\section{Fatores condicionantes da adaptação às mudanças climáticas na agricultura}

Grande parte dos trabalhos destinados a abordar a temática da adaptação às mudanças climáticas direcionam seus esforços em entender e modelar o

1 Entende-se por políticas públicas universais aquelas que possuem um único documento para ser seguido em todo o país. 
processo e as consequências das decisões tomadas pelos agricultores. Essa tarefa é importante na medida em que possibilita que os esforços realizados em termos da adaptação tenham sucesso e sejam eficientes naquilo que se propõem. Entender os determinantes das escolhas dos agricultores entre adaptar-se ou não, e conhecer o processo adjacente à tomada de decisão, é essencial para que políticas públicas sejam delineadas corretamente.

No entanto, há uma lacuna em termos de estudos e modelos que forneçam integração formal entre variáveis socioeconômicas e psicológicas/ cognitivas (percepção e crença nas alterações climáticas, além de questões subjetivas, como o raciocínio/julgamento sobre o potencial de impacto negativo na produção agrícola) associadas ao mecanismo decisório (Edward-Jones, 2006). A literatura puramente econômica baseia-se na teoria normativa e na pressuposição de que as decisões são modeladas somente tendo em vista a busca pela maximização do lucro (Borges et al., 2015). Essas são as pressuposições básicas da Teoria da Utilidade Esperada, segundo a qual o agricultor analisará se o ganho de utilidade proveniente da adoção de um mecanismo de adaptação (que se constitua em uma prática nova e sobre a qual ele não tem familiaridade) excede o ganho de utilidade de não utilizá-la.

Não obstante, essas ideias não permitem capturar toda a extensão e complexidade inerente à tomada de decisão dos agricultores. Por essa razão, é essencial considerar a Teoria do Comportamento Planejado. Essa corrente de pensamento postula que o comportamento humano é construído em termos de condições essencialmente psicológicas e origina-se de suas intenções de realizar e concretizar um comportamento específico. Dessa forma, a tomada de decisão não teria como base apenas questões econômicas em si, mas também fatores que são relacionados à percepção, habilidades cognitivas, conhecimento e oportunidades que eventualmente possam surgir e favorecer o comportamento positivo no sentido de adaptar-se em virtude das alterações climáticas (Wauters et al., 2010). Portanto, sob a premissa da Teoria do Comportamento Planejado, a decisão quanto à prática de ações adaptativas diz respeito à atitude que os agricultores têm frente às mudanças climáticas, às normas subjetivas e ao controle comportamental percebido que se origina, principalmente, das crenças do agricultor em questão (Hansson et al., 2012).
Diversos estudos vêm destacando que variáveis socioeconômicas tais como nível de escolaridade, o percentual da renda advinda do manejo da propriedade, tamanho da família, idade do produtor e a assistência técnica são determinantes do comportamento dos agricultores em termos da adoção de estratégias de adaptação. Além disso, podem ter efeito positivo e constituírem-se em incentivo para a utilização de novas tecnologias menos nocivas ao meio ambiente (Gebrehiwot \& Venn, 2013; Zeleke \& Aberra, 2014; Mabe et al., 2014; Galdies et al., 2016; Menike \& Arachchi, 2016; Niles \& Mueller, 2016; Tesfahunegn et al., 2016). Ademais, variáveis relativas à percepção, crença e conhecimento no que tange às mudanças climáticas e suas implicações sobre o produtor e propriedade são condições igualmente importantes e determinantes para a decisão de adaptação (Kpadonou et al., 2012; Lebel, 2013; Naess, 2013; Nguyen et al.,2016).

Problema de pesquisa semelhante ao do presente estudo já foi abordado por Deressa et al. (2011). Com o intuito de avaliar os fatores que determinam a percepção climática de agricultores da bacia do Nilo na Etiópia e se essa percepção traduz-se em adaptação frente às mudanças climáticas, os autores utilizaram o modelo de seleção amostral de Heckman. Os resultados evidenciaram que a percepção climática é relacionada à idade dos chefes da família, saúde, conhecimento das mudanças climáticas, capital social e a um conjunto de variáveis agroecológicas. Ademais, os principais fatores decisivos para a adaptação dizem respeito ao nível de escolaridade, tamanho da família, se o chefe da família é do sexo masculino, se o mesmo é proprietário do estabelecimento agropecuário e disponibilidade de crédito.

Mandleni \& Anim (2011) investigaram a extensão da conscientização sobre as mudanças climáticas de agricultores da província de Cabo Oriental na África do Sul. Para atingir os objetivos os autores utilizaram o modelo Probit. Os resultados indicaram que o nível de educação, o estado civil, a temperatura e a forma como a terra foi adquirida afetam significativamente o conhecimento sobre as mudanças climáticas.

Temesgen et al. (2014) estudaram como os agricultores do sudeste da Etiópia percebem as mudanças climáticas, quais tipos de mecanismos de adaptação tais agricultores utilizam e que fatores influenciam a adaptação às mudanças climáticas. Para acessar essas questões os autores utilizaram o modelo Logit Multinomial e constataram que há uma associação forte e positiva entre a combinação de práticas 
agronômicas e o uso de insumos agrícolas com o nível educacional, acesso à informação, acesso a crédito e renda do estabelecimento. Similarmente, o sexo do chefe da família e o acesso à informação afetaram positivamente a decisão de adaptação desses agricultores. Temesgen et al. (2014) dão destaque à necessidade de melhorias quanto ao acesso a mecanismos de crédito e da promoção da educação à adultos para que os agricultores da região possam utilizar medidas de adaptação eficientes e que os permita anteverem-se aos impactos adversos do clima.

Estudos que consideram explicitamente o papel do conhecimento sobre mudanças climáticas no investimento em estratégias adaptativas como os que foram descritos anteriormente ainda não são difundidos no Brasil. No entanto, questões sobre percepção/ previsão climática e adaptação têm sido abordadas por diferentes autores, tais como Lemos et al. (2002), Obermaier \& Rosa (2013), Pires et al. (2014), Andrade et al. (2014), Fuentes et al. (2015). Todos esses estudos, exceto o de Pires et al. (2014), abordam essa problemática no contexto do Nordeste, que é, conforme já mencionado, a região brasileira para a qual são esperados os maiores impactos negativos no setor agrícola.

Lemos et al. (2002) analisaram como as previsões climáticas são utilizadas pelo poder público para reduzir efeitos adversos da seca no Ceará. Conforme os autores, embora as previsões sazonais ofereçam oportunidades importantes de comportamento proativo contra a seca, sua eficácia, à época da análise, ainda era limitada e inadequada para o desenvolvimento de políticas voltadas para a agricultura. Foram detectados erros de interpretação das informações, bem como indícios de manipulação política.

Andrade et al. (2014) estudaram a percepção sobre mudanças climáticas e as estratégias de adaptação de agricultores da região conhecida como Seridó, no Rio Grande do Norte. Os resultados da pesquisa indicaram que

(...) a maior parte dos agricultores percebeu alterações na estação chuvosa (chuvas mais fortes), na temperatura (clima mais quente), redução da ocorrência de anos secos e maior imprevisibilidade e/ ou deslocamento dos meses quentes e frios (Andrade et al., 2014, p. 94).

Ademais, segundo os autores, as estratégias adaptativas adotadas estavam diretamente relacionadas à cultura local de enfrentamento das secas. Resultado semelhante foi obtido por Fuentes et al. (2015), ao estudarem produtores rurais do semiárido do estado da Bahia. Entre as principais conclusões, Fuentes et al. (2015, p. 362) destacam que, devido ao fato de a área estudada pertencer ao Polígono das Secas, a observação de sinais da natureza indicativos de chuva constitui-se "uma estratégia secular do homem do campo para se adaptar à variabilidade climática local".

De acordo com Obermaier \& Rosa (2013), a identificação de fatores de redução da vulnerabilidade às mudanças do clima de agricultores, sobretudo em regiões semiáridas, é fundamental para aumentar a resiliência do setor agrícola. Nesse contexto, os autores concluem que o fato de grande parte da população rural do semiárido nordestino já estar sofrendo grandes impactos negativos implica que "(...) ações para reduzir sua vulnerabilidade não dependem necessariamente da certeza de futuros impactos climáticos" (Obermaier \& Rosa, 2013, p. 170). Argumentação semelhante é apresentada por Pires et al. (2014). Segundo esses autores, em Minas Gerais, embora os produtores tenham conhecimento sobre mudanças climáticas e estejam percebendo alterações nos padrões de precipitação, as respostas em termos de ações adaptativas ainda são limitadas, o que pode levar a maior nível de vulnerabilidade. Lemos \& Kirchhoff (2016) explicam que frequentemente há desconexão entre as informações disponíveis e a capacidade de adaptação. Ao mesmo tempo, Andrade et al. (2014) argumentam que muitas vezes a adaptação não ocorre devido à falta de informações sobre as estratégias adequadas / disponíveis e à baixa capacidade adaptativa.

Dessa forma, pode-se afirmar que estudos realizados para o Brasil indicam que a percepção de alterações esperadas do clima é importante para as ações de adaptação, mas deve ser pensada em conjunto com variáveis socioeconômicas que impliquem em maior capacidade adaptativa. Não obstante, a literatura que versa sobre a percepção e adaptação às mudanças climáticas destaca que o conhecimento que os agricultores têm em termos do fenômeno e de seus impactos sobre o manejo e condução das atividades em seus estabelecimentos aliada à forma como interpretam e gerenciam as informações climáticas disponíveis são um pré-requisito fundamental para a adoção de mecanismos de adaptação (Blennow \& Persson, 2009; Below et al., 2012; Blennow et al., 2012; Haden et al., 2012; Hagen, 2016). Por fim, Nguyen et al. (2016) ressaltam que o conhecimento molda a percepção climática dos agricultores que, por sua vez, molda a decisão de adoção de medidas adaptativas. Os autores destacam que outras variáveis também têm papel decisivo 
sobre a escolha do agricultor, mas são categóricos ao afirmar que conhecimento, percepção e adaptação são variáveis altamente correlacionadas.

\section{Metodologia}

\subsection{Probit Bivariado Aparentemente Não Relacionado}

O objetivo principal deste estudo é analisar se o conhecimento de um agricultor sobre o fenômeno das mudanças climáticas e a adoção de mecanismos de adaptação são fenômenos interligados. Para atender a tal objetivo foi utilizada a técnica conhecida como Probit Bivariado Aparentemente Não Relacionado. Esse modelo empírico foi utilizado para levar em conta o fato de que as variáveis que afetam o conhecimento e a adaptação são correlacionadas, levando à obtenção de estimativas inconsistentes dos parâmetros. Ademais, o conhecimento e a adaptação também se correlacionam entre si; conhecimento possibilita que o agricultor se adapte e, uma vez que ele tenha se adaptado, o aprendizado gerado pelo mecanismo de adaptação leva a maior conhecimento (simultaneidade) ${ }^{2}$.

O modelo Probit Bivariado Aparentemente Não Relacionado apresenta duas variáveis dependentes discretas de interesse (Cameron \& Trivedi, 2005). As variáveis dependentes consideradas foram "Conhece" - variável dummy que assume valor igual a 1 quando o agricultor já ouviu falar sobre as mudanças climáticas, acredita na sua ocorrência e nos impactos negativos que podem recair sobre sua propriedade -, e "Adaptou-se" - variável dummy que assume valor igual a 1 quando o agricultor em questão alterou o manejo de seu estabelecimento agrícola em virtude das alterações climáticas.

Segundo Cameron \& Trivedi (2005), a principal característica do modelo utilizado nesta pesquisa está relacionada à especificação da forma funcional para as probabilidades, as quais devem considerar a correlação de

2 Considerando a discussão neste estudo, pode-se afirmar que é teoricamente e metodologicamente errôneo estimar um modelo de resposta qualitativa univariado (Logit ou Probit) para avaliar o papel do conhecimento climático sobre a decisão de adaptação dos agricultores. Isso, pois, seria necessário estimar um modelo para avaliar os determinantes do conhecimento e outro para verificar os fatores que impactam a decisão de adaptação, o que levaria à obtenção de estimativas inconsistentes dos parâmetros. dois resultados e, possivelmente, a simultaneidade. Dessa forma, a probabilidade de que um agricultor conheça o fenômeno das mudanças climáticas e adapte-se, pode ser expressa como segue:

$$
p_{i j k}=\operatorname{Pr}[\text { Conhece }=j, \text { Adaptou }=k], j=0 \text { ou } 1 \text { e } k=0 \text { ou } 1
$$

Note que essa probabilidade define um conjunto de eventos mutuamente exclusivos, de modo que:

$\sum_{j k} \sum_{i j k}=1$

Sejam as variáveis latentes não observáveis expressas nas equações (3) e (4):

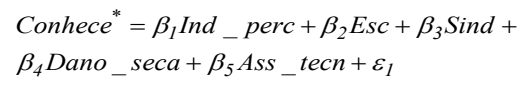

em que a variável "Ind _ perc" designa o Índice de Percepção Climática ${ }^{3}$ dos agricultores da bacia do Rio das Contas; "Esc" é o nível de escolaridade desses agricultores; "Sind" refere à participação em algum tipo de associação de classe ou sindicato rural; "Dano_seca" é o risco que o respectivo agricultor atribui a perdas financeiras em virtude de secas na região; e "Ass_tecn" evidencia se o agricultor recebeu assistência técnica.

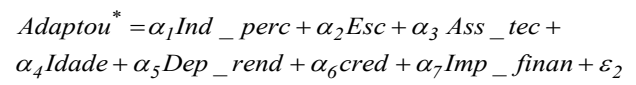

em que a variável "Ind_perc" designa o Índice de Percepção Climática dos agricultores da bacia do Rio das Contas; "Esc" é o nível de escolaridade desses agricultores; "Ass_tecn" evidencia se o agricultor recebeu assistência técnica; "Dep_ren" é uma variável dummy que assume valor igual a 1 se o produtor tem a totalidade da sua renda oriunda do estabelecimento agrícola e, além disso, não desempenha atividade diferente da agricultura para obtenção de renda; "cred" diz respeito ao fato do agricultor ter acesso a crédito para desenvolver suas atividades; e, por fim, "Imp_finan" é uma variável que mensura se o agricultor entrevistado acredita que as

3 Para compreender a percepção dos agricultores em termos das mudanças climáticas, foram consideradas as respostas dos entrevistados em relação à (i) observação de algum tipo de evento extremo que possa ser atribuído às mudanças climáticas; (ii) observação de alteração da temperatura e precipitação de verão e inverno tendo como base o ano de 2013; ou seja, é a percepção presente que aqueles agricultores têm. O índice foi construído utilizando Análise Fatorial por Componentes Principais, com base na proposição metodológica de Filmer e Pritchett (2001). Detalhes sobre o cálculo do índice são apresentados no Anexo. Índice de percepção climática. 
Figura 1. Bacia hidrográfica do Rio das Contas e sua localização no estado da Bahia e no Brasil

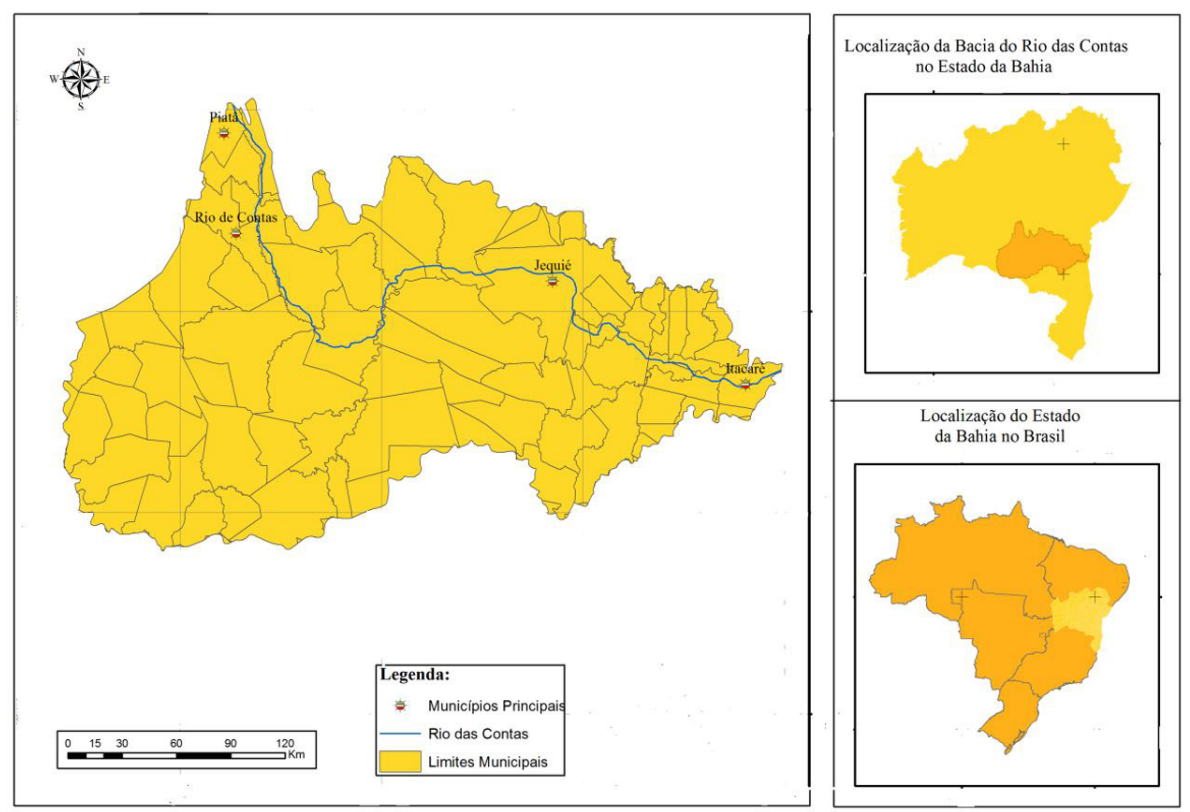

Fonte: Elaboração própria com base nos dados do Instituto Brasileiro de Geografia e Estatística (2010).

mudanças climáticas afetarão a situação financeira de sua propriedade.

Cabe ressaltar que $\varepsilon_{1}$ e $\varepsilon_{2}$ presentes nas equações (3) e (4) são conjuntamente normalmente distribuídos com média zero, variância um e correlação $\rho$, ou seja:

$\left(\begin{array}{l}\varepsilon_{1} \\ \varepsilon_{2}\end{array}\right) \sim N\left\{\left(\begin{array}{l}0 \\ 0\end{array}\right),\left[\begin{array}{ll}1 & \rho \\ \rho & 1\end{array}\right]\right\}$

Dessa forma, o modelo Probit Bivariado especifica os resultados como sendo:

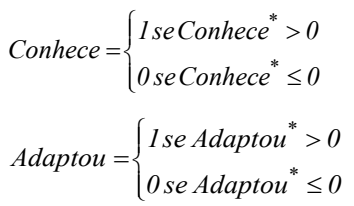

Esse modelo colapsa em dois modelos Probit distintos se a correlação entre os resíduos das equações estimadas são independentes $(\rho=0)$. Quando $\rho \neq 0$, não há uma solução fechada para as probabilidades (Cameron \& Trivedi, 2005). Dessa forma, as equações não podem ser estimadas isoladamente.

Devido ao fato de as equações destacadas serem aparentemente não correlacionadas e das variáveis dependentes consideradas serem ambas discretas, optou-se por estimá-las através de um modelo Probit Bivariado Aparentemente Não Relacionado. Segundo Baum (2006), a estimação desse tipo de modelo é semelhante à de um modelo de Regressões Aparentemente Não Relacionadas (SUR). A principal vantagem da especificação dos modelos SUR é seu tratamento da correlação entre observações num ponto particular do tempo (Moon \& Perron, 2008).

\subsection{Dados e área de estudo}

Para a avaliação do conhecimento, percepção e do comportamento dos produtores rurais da bacia do Rio das Contas em relação às mudanças climáticas foi aplicado um questionário ${ }^{4}$ semiestruturado englobando questões relacionadas às (i) informações socioeconômicas dos produtores e de suas propriedades (tais como, experiência, idade, escolaridade e acesso a meios de informação); (ii) percepção desses produtores em relação às mudanças do clima; e (iii) medidas adaptativas implementadas nas propriedades. Os questionários foram aplicados entre setembro e novembro de 2014 em 26 municípios que fazem parte da bacia do Rio das Contas (Figura 1).

4 Ressalta-se que esses dados primários foram coletados no âmbito de um projeto financiado pelo Conselho Nacional de Desenvolvimento Científico e Tecnológico-CNPq (Processo 408382 / 2013-9) e pela Fundação de Amparo à Pesquisa do Estado de Minas Gerais - Fapemig (Processo CSA-PPM-00022-14). A aplicação dos questionários foi aprovada pelo Comitê de Ética em Pesquisa com Seres Humanos da Universidade Federal de Viçosa (CAAE: 30752814.2.0000.5153). 
Seguindo a metodologia proposta por Hartter (2009) e Blennow et al. (2012), do universo de 145.647 estabelecimentos agrícolas dos 92 municípios que compõem a bacia (Instituto Brasileiro de Geografia e Estatística, 2006), foi selecionada uma amostra de 289 propriedades rurais por amostragem aleatória simples com 95\% de confiança. O desenho amostral foi criado de forma a obter distribuição aleatória de propriedades agrícolas através da grande variedade de zonas climáticas dentro da região que compreende a bacia do Rio das Contas. Conforme a tipologia climática de Köppen-Geiger (Maksoud, 1964; Superintendência de Estudos Econômicos e Sociais da Bahia, 1998; Peel et al., 2007), a bacia do Rio das Contas compreende o clima tropical chuvoso de floresta, caracterizado pela ausência de estação seca, pluviosidade média mensal superior a $60 \mathrm{~mm}$ e anual superior a $1.500 \mathrm{~mm}$, temperatura do mês mais frio acima de $18^{\circ} \mathrm{C}$ e veróes longos e quentes com temperatura do mês mais quente superior a $22^{\circ} \mathrm{C}$; o clima seco (típico de caatinga), cuja principal característica é a ausência de excedente hídrico, é acompanhada pela predominância de chuvas de verão e período seco bem definido de inverno e temperatura média superior a $18^{\circ} \mathrm{C}$; e clima tropical de altitude, com predominância de chuvas de verão e seca de inverno, temperatura do mês mais frio superior a $18^{\circ} \mathrm{C}$, temperatura do mês mais quente superior a $22^{\circ} \mathrm{C}$ e altitude superior a $1200 \mathrm{~m}$.

De acordo com as especificações da metodologia de Hartter (2009), criou-se um "buffer zone" (Figura 2) que englobou municípios localizados, no máximo, a $50 \mathrm{~km}$ de toda a extensão do Rio das Contas. A "buffer-zone" cobriu a área de estudo no sentido Leste-Oeste e, por meio dela, selecionou-se, aleatoriamente, as coordenadas geográficas das propriedades consideradas no estudo.

O número de questionários aplicados em cada município foi proporcional ao número total de produtores do município em questão, tendo como base as informações disponibilizadas pelo Censo Agropecuário (Instituto Brasileiro de Geografia e Estatística, 2006). A amostragem teve ainda um nível de estratificação, referente à propriedade da terra. Assim, o número de produtores entrevistados em cada município foi proporcional ao

Figura 2. "Buffer-zone" para a seleção dos municípios amostrados

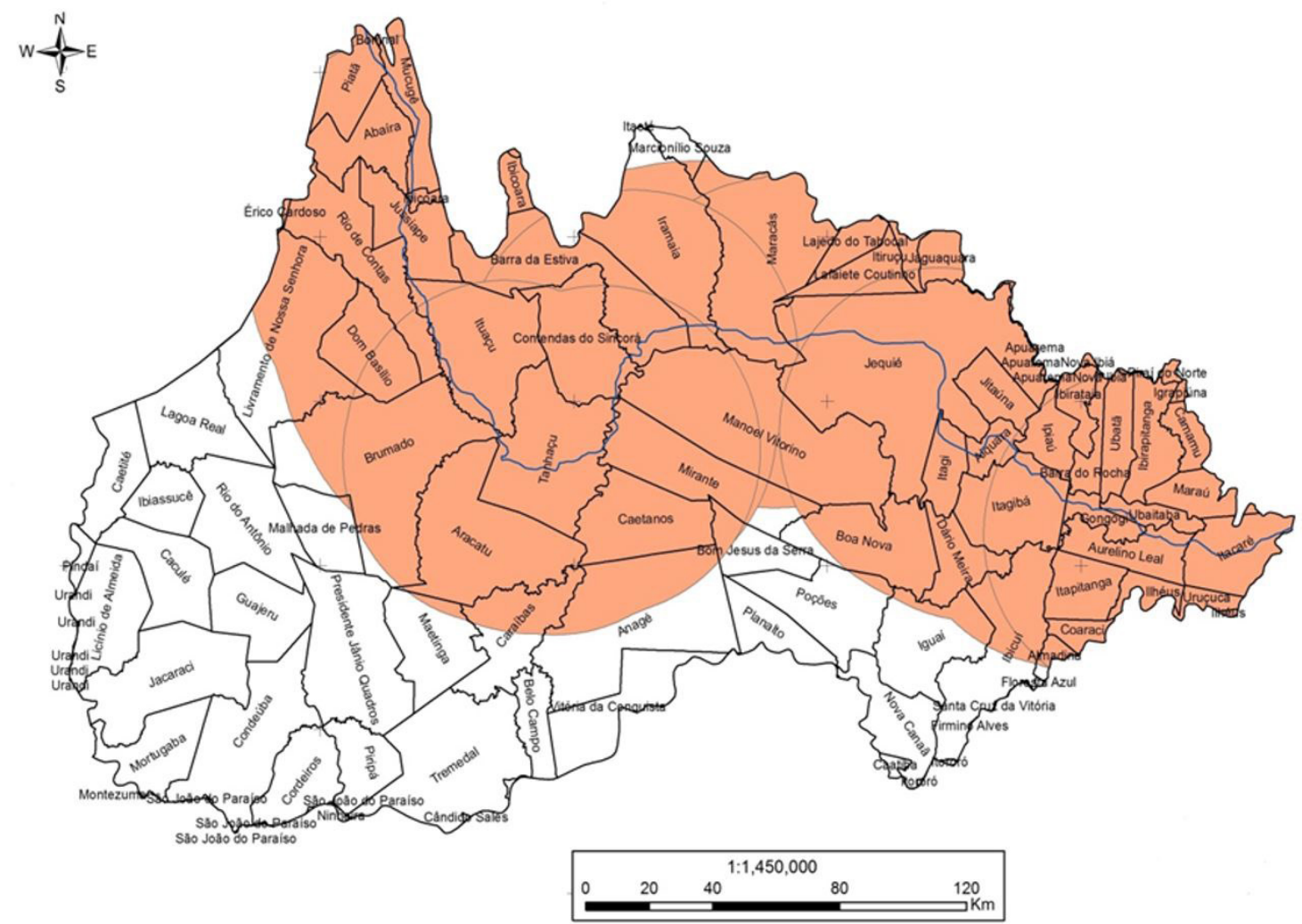

Fonte: Elaboração própria com base nos dados do Instituto Brasileiro de Geografia e Estatística (2010) e na metodologia de Hartter (2009). 
número de proprietários e não proprietários (parceiros, meeiros, arrendatários, posseiros etc.) disponibilizados pelo Censo Agropecuário (Instituto Brasileiro de Geografia e Estatística, 2006).

\section{Resultados}

Os dados coletados pela pesquisa indicam que, em média, 52\% dos agricultores entrevistados alteraram o manejo da sua propriedade devido aos eventos climáticos, ou seja, adaptaram-se. Com relação ao conhecimento sobre as mudanças climáticas, constata-se que aproximadamente $69 \%$ dos agricultores já ouviu falar sobre as mudanças climáticas, acredita na sua ocorrência e compreende os impactos negativos que podem recair sobre sua propriedade. Esses valores relativamente altos para conhecimento e adaptação possivelmente são resultado das experiências passadas vividas pelos agricultores. Conforme indicam dados históricos fornecidos pelo Climate Research Unit (2013), a região da bacia do Rio das Contas tem passado por reduções expressivas de seus índices de precipitação e aumentos nos valores médios de temperatura nos últimos trinta anos. Ademais, segundo Marengo et al. (2017), há evidências meteorológicas para apoiar a percepção dos agricultores sobretudo no que diz respeito às mudanças dos padrões históricos de precipitação, já que o Nordeste brasileiro tem enfrentado, desde 2012, a pior seca dos últimos 100 anos.

Dados sobre as estatísticas descritivas das variáveis explicativas consideradas neste estudo são apresentadas na Tabela 1. Ainda que de modo preliminar, os dados da Tabela 1 permitem fazer importantes considerações com relação à adoção de práticas adaptativas e o conhecimento sobre as mudanças climáticas pelos agricultores da bacia do Rio das Contas, Bahia.

De modo geral, observa-se que os agricultores que se adaptaram em virtude das mudanças climáticas e conhecem e acreditam na ocorrência do fenômeno perceberam mais alterações nas temperaturas de verão e inverno e presenciaram algum tipo de evento extremo que atribuíram como sendo decorrente das mudanças climáticas. Além disso, aqueles que se adaptaram e conhecem o fenômeno acreditam que terão maiores impactos negativos do que aqueles que não se adaptaram e desconhecem o problema. Grande parte dos agricultores também considera que terá risco alto ou muito alto de ser prejudicado financeiramente pela seca, sendo que $82 \%$ daqueles que se adaptaram têm adotado principalmente a irrigação como medida adaptativa para reduzir esse risco (Tabela 1).

Tabela 1. Estatísticas descritivas das variáveis utilizadas

\begin{tabular}{|c|c|c|c|c|c|c|c|}
\hline \multirow[b]{2}{*}{ Variável } & \multicolumn{4}{|c|}{ Média } & \multirow{2}{*}{$\begin{array}{l}\text { Desvio } \\
\text { Padrão }\end{array}$} & \multirow[b]{2}{*}{ Mínimo } & \multirow[b]{2}{*}{ Máximo } \\
\hline & Adaptou & $\begin{array}{c}\text { Não } \\
\text { adaptou }\end{array}$ & Conhece & $\begin{array}{c}\text { Não } \\
\text { conhece }\end{array}$ & & & \\
\hline Índice de percepção climática & 0,76 & 0,69 & 0,77 & 0,64 & 0,19 & 0 & 1 \\
\hline Impacto financeiro decorrente & 5,00 & 4,00 & 5,00 & 4,00 & 1,00 & 1 & 5 \\
\hline Dano causado pela seca & 0,82 & 0,75 & 0,85 & 0,64 & 0,40 & 0 & 1 \\
\hline Idade & 51 & 50 & 50 & 52 & 15,38 & 18 & 93 \\
\hline Escolaridade & 4,00 & 2,00 & 3,00 & 2,00 & 2,06 & 0 & 7 \\
\hline Dependência de renda & 0,48 & 0,40 & 0,43 & 0,49 & 0,49 & 0 & 1 \\
\hline Crédito & 0,32 & 0,20 & 0,31 & 0,17 & 0,44 & 0 & 1 \\
\hline Assistência técnica & 0,39 & 0,20 & 0,35 & 0,17 & 0,45 & 0 & 1 \\
\hline Sindicato & 0,68 & 0,60 & 0,71 & 0,48 & 0,44 & 0 & 1 \\
\hline
\end{tabular}

Fonte: Dados da pesquisa.

Notas: A variável "Impacto financeiro decorrente" diz respeito à crença dos agricultores considerados quanto ao impacto financeiro que irá recair sobre suas propriedades em virtude das mudanças climáticas. Na média, os agricultores que se adaptaram e conhecem o fenômeno acreditam que serão impactos de forma muito negativa (Alternativa 5). Todavia, aqueles que não se adaptaram e não conhecem o fenômeno acreditam que serão afetados mais ou menos negativamente (Alternativa 4). A variável "Danos causados pela seca" é uma variável dummy que assume valor igual a 1 quando o agricultor acredita que sua produção e propriedade terá um risco alto ou muito alto de ser impactado negativamente pela seca e 0 caso contrário. A variável "Escolaridade" é uma variável categórica que assume valores entre 0 e 7 de acordo com o nível de escolaridade de cada agricultor entrevistado. Aqueles que se adaptaram têm, em média, o Ensino Médio incompleto (Alternativa 4), enquanto que aqueles que não se adaptaram e não conhecem o fenômeno das mudanças climáticas têm, em média, o Ensino Fundamental incompleto (Alternativa 2). Aqueles que conhecem o fenômeno têm, em média, o Ensino Fundamental completo (Alternativa 3). 
A variável "Idade", aqui utilizada como proxy da experiência dos agricultores, não diferiu consideravelmente entre aqueles que se adaptaram ou não e aqueles que conhecem ou não as mudanças climáticas. Todavia, observa-se na Tabela 1 que o nível educacional tem considerável importância para explicar a adaptação e o conhecimento. Aqueles que optaram por modificar o manejo de sua propriedade e creem na ocorrência das mudanças climáticas têm maiores níveis educacionais.

Outra característica importante evidenciada pela análise estatística dos dados é que a dependência de renda oriunda da propriedade, pelo menos a princípio, parece não ser fator determinante da opção pela adoção de práticas adaptativas. Com relação ao acesso a crédito e à assistência técnica, a Tabela 1 evidencia que a maioria dos agricultores considerados não tem acesso a nenhum dos dois serviços. Além disso, observa-se que, em média, os produtores com maior grau de percepção sobre mudanças climáticas e que adotaram práticas adaptativas têm maior participação em associações de classe ou sindicato rural.

Feitas essas considerações sobre as variáveis, pode-se discutir a relação entre o conhecimento sobre as mudanças climáticas e seus impactos sobre a propriedade, bem como a percepção climática dos agricultores e a decisão de adaptação. Para avaliar se conhecimento e adaptação são questões que devem ser analisadas em conjunto em estudos que têm como objetivo discutir ou pensar em adoção de práticas adaptativas foi estimado um modelo Probit Bivariado Aparentemente Não Relacionado. Os resultados da estimação encontram-se descritos na Tabela 2. A hipótese testada nas análises apresentadas a seguir é que o conhecimento exerce grande influência sobre o planejamento adaptativo. Em outras palavras, os agricultores tomam suas iniciativas com base no conhecimento que julgam ter a respeito do fenômeno e de suas implicações sobre sua produção e propriedade.

O teste de razão de verossimilhança confirmou que os resíduos das equações consideradas não são independentes $(\rho=0,50$ e p-valor $<0,00)$. Dessa forma, seria errôneo estimá-las separadamente. Esse resultado indica que conhecimento e adaptação são variáveis correlacionadas e não considerar essa relação em estudos que versam sobre adaptação pode levar a estimativas viesadas dos parâmetros. De acordo com Kpadonou et al. (2012), as comunidades locais optam por implementar

Tabela 2. Estimativas do modelo Probit Bivariado Aparentemente Não Relacionado sobre o conhecimento e adoção de medidas adaptativas pelos produtores da bacia do Rio das Contas

\begin{tabular}{|c|c|c|}
\hline Variável & Coeficiente & P-valor \\
\hline \multicolumn{3}{|l|}{ Conhece } \\
\hline Índice de percepção climática & $1,57^{* * *}$ & 0,00 \\
\hline Dano causado pela seca & 0,36 & 0,11 \\
\hline Escolaridade & $0,15^{* * *}$ & 0,00 \\
\hline Assistência técnica & $0,51^{* * *}$ & 0,01 \\
\hline Sindicato & $0,38^{* *}$ & 0,03 \\
\hline Constante & $-1,69^{* * *}$ & 0,00 \\
\hline \multicolumn{3}{|l|}{ Adaptou-se } \\
\hline Índice de percepção climática & $1,17^{* * *}$ & 0,01 \\
\hline Impacto financeiro decorrente & 0,06 & 0,51 \\
\hline Idade & $0,06^{* * *}$ & 0,00 \\
\hline Escolaridade & $0,24^{* * *}$ & 0,00 \\
\hline Dependência da renda gerada pela propriedade & $0,003^{*}$ & 0,10 \\
\hline Crédito & 0,25 & 0,16 \\
\hline Assistência Técnica & $0,63^{* * *}$ & 0,00 \\
\hline Constante & $-3,06^{* * *}$ & 0,00 \\
\hline \multicolumn{3}{|l|}{$N=289$} \\
\hline \multicolumn{3}{|l|}{$\rho=0,50$} \\
\hline Teste da razão de verossimilhança de $\rho=0$ & $\chi^{2}=21,37^{* * *}$ & 0,00 \\
\hline
\end{tabular}

Fonte: Dados da pesquisa. Notas: $\left(^{(* *)},\left(^{(*)}\right.\right.$ e $\left(^{*}\right)$ indicam significância a 1\%,5\% e 10\%, respectivamente. 
estratégias de adaptação tendo em vista seus recursos e, principalmente, suas relações sociais informais e seus valores que, por sua vez, traduzem-se em seu conhecimento a respeito do fenômeno. Ademais, segundo Lebel (2013), o conhecimento e crença nas mudanças climáticas não são importantes somente porque se traduzem na adoção de práticas adaptativas, mas também porque permitem, por meio da interação entre os agentes envolvidos, que mais conhecimento seja gerado e novas técnicas e mecanismos adaptativos sejam descobertos e utilizados.

A análise da Tabela 2 indica também que as variáveis "Índice de percepção climática", "Escolaridade" e "Assistência técnica" foram positivamente correlacionadas e estatisticamente significativas para explicar o conhecimento e a escolha pela adaptação. Segundo Tesfahunegn et al. (2016), a percepção das mudanças climáticas bem como o nível de escolaridade e assistência técnica influenciam o sucesso das estratégias de adaptação e das práticas de manejo da propriedade.

A percepção climática também se mostrou importante e determinante da decisão de adaptação em estudos tais quais os de Abid et al. (2015), Galdies et al. (2016), Menike \& Arachchi (2016), Niles \& Mueller (2016) e Li et al. (2017). De forma geral, esses estudos destacam que a percepção de que o clima está se alterando e dos impactos que podem ser sentidos em termos local e global são parte fundamental do processo de decisão quanto à adaptação ou não adaptação. Além disso, o nível de percepção determinará se a resposta frente a esses eventos será efetiva e eficiente. A percepção climática aliada ao conhecimento e maior disponibilidade de informações climáticas possibilita a redução dos riscos inerentes às alterações climáticas e eleva as chances da redução dos impactos negativos que podem recair sobre os agricultores e suas propriedades.

A escolaridade e assistência técnica são importantes na medida em que permitem a redução dos riscos relacionados aos eventos extremos. Esses dois fatores têm efeito direto sobre o conhecimento de estratégias, mecanismos e tecnologias que são capazes de promover a redução da vulnerabilidade a eventos adversos do clima. Ademais, possibilitam que os agentes envolvidos tenham mais condições de escolher entre as diferentes possibilidades de estratégias adaptativas disponíveis, minimizando possíveis perdas (Wamsler et al., 2012).

Os resultados evidenciaram ainda que o fato de o agricultor ser associado a um sindicato rural e de acreditar que os danos causados pela seca serão altos ou muito altos sobre sua produção e propriedade são positivamente correlacionados ao conhecimento. No entanto, a variável "Dano causado pela seca" não foi estatisticamente significativa. É importante destacar que o fato de ser associado a um algum tipo de cooperativa ou entidade de classe permite que os produtores tenham maior acesso a informações sobre a utilização de determinada técnica ou tecnologia, além de possibilitar o compartilhamento de experiências e conhecimentos.

Com relação à adaptação, observa-se que a idade do agricultor e a dependência da renda gerada pelo manejo da propriedade foram estatisticamente significativos e positivamente correlacionados à escolha pela adoção de medidas adaptativas frente às mudanças climáticas.

O efeito da variável "Idade" sobre a decisão de adaptar-se diverge na literatura. Enquanto alguns estudos, como o de Uddin et al. (2014), evidenciam que a idade dos agricultores é negativamente relacionada a decisão de adaptação, outros, como o de Deressa et al. (2011) e Etwire et al. (2013), destacam sua importância e efeito positivo sobre o processo decisório. Dessa forma, acredita-se que a variável "Idade" tem impacto sobre o conhecimento, experiência e, consequentemente, sobre a decisão de adaptação. Agricultores com maior idade têm mais conhecimento acerca do manejo da propriedade e maior experiência em práticas agrícolas que permitem reduzir os efeitos negativos das mudanças climáticas. Além disso, mais experiência no manejo do seu estabelecimento possibilita maior percepção climática e, consequentemente, aumenta probabilidade de adoção de estratégias de adaptação (Deressa et al., 2011; Mudzonga, 2012; Etwire et al., 2013; Debela et al., 2015).

O efeito da variável "Dependência da renda gerada" foi pequeno sobre a decisão de adaptação. Esse resultado pode estar evidenciado que a atividade agrícola não é percebida unicamente como geradora de renda, mas como algo que é intrínseco à cultura da região e dos agricultores. Dessa forma, as ações dos agricultores considerados diante das mudanças climáticas podem ser motivadas também por valores simbólicos, não captados explicitamente pelo instrumento de coleta de dados e que são representados pela vontade de permanecer na terra ou no local de origem.

As variáveis "Crédito" e "Impacto financeiro decorrente" foram positivamente correlacionadas à adaptação, porém, não foram estatisticamente significativas. $\mathrm{O}$ acesso a 
linhas de crédito é um fator importante na medida em que permite que o produtor utilize mecanismos que reduzam os riscos advindos das mudanças climáticas, além de novas tecnologias que possibilitam alcançar eficiência produtiva e ambiental. Contudo, a maior parte dos agricultores considerados (73\%) não teve acesso à nenhuma modalidade de crédito e esse pode ser um dos fatores para explicar a não significância dessa variável na decisão de adaptação. O que se observa é que muitos dos agricultores entrevistas não dispõem de conhecimentos acerca da concessão de crédito, são submetidos à grande burocracia para ter acesso ao financiamento e também são avessos ao risco de incorrer em tais sistemas crédito. Essa é uma questão que merece maior atenção por parte dos formuladores de políticas públicas, uma vez que toda a política de concessão de crédito que não leve em consideração os problemas e incertezas impostas aos agricultores está fadada ao insucesso.

Outro fator relevante e que pôde ser observado por meio da análise dos resultados é que, de forma geral, os produtores entrevistados acreditam na ocorrência das mudanças climáticas, mas veem o fenômeno como algo ainda distante de sua realidade. Entretanto, esses agricultores já observam impactos advindos das mudanças no clima, como a seca e a redução dos níveis de precipitação. Essa aparente contradição pode estar evidenciando a razão pela qual a variável "Impacto financeiro decorrente" não foi estatisticamente significativa para explicar a opção pela adaptação.

\section{Conclusão}

Os resultados obtidos evidenciaram que conhecimento e adaptação são correlacionados e não devem ser pensados de forma isolada. Observou-se que os agricultores que conhecem os efeitos das mudanças climáticas sobre suas atividades agrícolas são os que possuem maior probabilidade de adotar medidas adaptativas. Isso reforça a ideia de que, em análises de adaptação, deve ser considerado o papel do conhecimento dos atores envolvidos. Portanto, políticas como o Plano Nacional de Adaptação, instituído pelo governo federal em 2016, deveriam ser precedidas por ações educativas que tenham como meta aumentar o conhecimento acerca das alterações futuras do clima aos agricultores. Isso é de extrema importância, pois, muitas vezes, o agricultor tem percepção sobre as mudanças climáticas, mas nem sempre possui o conhecimento adequado de açóes que podem ser empreendidas para lidar com o fenômeno.

Outra questão que merece destaque é a necessidade de maior acesso à assistência técnica, a qual tem potencial de capacitar os produtores a adotarem estratégias de adaptação e mitigação. Nesse sentido, políticas públicas que visem ampliar a adoção de mecanismos de adaptação na região devem promover maior acesso ao serviço, uma vez que $70 \%$ dos produtores considerados neste estudo não tem acesso a ele.

Os resultados encontrados também evidenciaram que as atividades agrícola e pecuária não são tidas apenas como fonte de renda, mas como algo intrinsecamente associado à cultura dos agricultores. Dessa forma, a tomada de decisão também é pautada na vontade de permanecer na terra e na disponibilidade de recursos naturais.

As discussões aqui desenvolvidas reforçam a necessidade de políticas públicas voltadas à maior divulgação de informações climáticas regionais, aliada a políticas que ampliem o acesso à assistência técnica ou extensão rural. Além disso, chama-se atenção para a importância de se considerar, ainda nas etapas iniciais do processo de formulação da política, a percepção, o conhecimento e as condições socioeconômicas dos indivíduos envolvidos. Essas são considerações fundamentais para o sucesso e eficiência dos esforços das políticas públicas regionais.

As políticas devem ser desenvolvidas no sentido de promover e disseminar estratégias que guardem alguma relação com a realidade dos agricultores. Além disso, devem considerar o conhecimento que os agricultores dispõem sobre o assunto, já que esse fator será determinante para o sucesso da estratégia delineada. Apesar de algumas dessas técnicas serem relativamente simples, podem ter impacto fundamental no sentido da redução e amenização dos efeitos do clima sobre a produção agrícola.

Por fim, é importante ressaltar que o presente estudo não é capaz de chegar a conclusões concretas sobre os determinantes das crenças pessoais no fenômeno das mudanças climáticas. Nesse sentido, sugere-se que estudos futuros testem a hipótese de que a experiência direta causa crença nas mudanças climáticas e a crença, por sua vez, desencadeia o processo adaptativo. Além disso, sugere-se a realização de estudos em outras regiões com características edafoclimáticas e condições socioeconômicas 
distintas, já que isso permitiria a construção de um panorama completo sobre os pontos fortes e fracos da adaptação de produtores rurais no Brasil.

\section{Agradecimentos}

O presente trabalho foi realizado com apoio da Coordenação de Aperfeiçoamento de Pessoal de Nível Superior - Brasil (CAPES) - Código de Financiamento 001. Os autores Dênis Antônio da Cunha e Sabrina de Matos Carlos agradecem ao Conselho Nacional de Desenvolvimento Científico e Tecnológico - CNPq pela concessão de Bolsa de Produtividade em Pesquisa (Processo 305807 / 2018-8 e 421791/2016-0) e Bolsa de Doutorado Pleno - GD, respectivamente.

\section{Referências}

Abid, M., Scheffran, J., Schneider, U. A., \& Ashfaq, M. (2015). Farmers' perceptions of and adaptation strategies to climate change and their determinants: the case of Punjab province, Pakistan. Earth System Dynamics, 6, 225-243.

Andrade, A. A. X., Silva, G. B., \& Andrade, N. A. X. (2015). $\mathrm{O}$ acesso às políticas públicas no semiárido e seu reflexo na heterogeneidade da agricultura familiar: o PNAE em Boquira - BA. Revista Extensão Rural, 22(2), 79-97.

Andrade, A. J. P., Silva, N. M., \& Souza, C. R. (2014). As percepções sobre as variações e mudanças climáticas e as estratégias de adaptação dos agricultores familiares do Seridó potiguar. Desenvolvimento e Meio Ambiente, 31, 77-96.

Baum, C. F. (2006). An introduction to modern econometrics using stata. College Station: Stata Press.

Below, T. B., Mutabazi, K. D., Kirschke, D., Franke, C., Sieber, S., Siebert, R., \& Tscherning, K. (2012). Can farmers' adaptation to climate change be explained by socioeconomic household-level variables? Global Environmental Change, 22, 223-235.

Blennow, K., \& Persson, J. (2009). Climate change: motivation for taking measure to adapt. Global Environmental Change, 19, 100-104.

Blennow, K., Persson, J., Tomé, M., \& Hanewinkel, M. (2012). Climate change: believing and seeing implies adapting. PLoS One, 7(11), e50182.

Borges, J. A. R., Foletto, L., \& Xavier, V. T. (2015). An interdisciplinary framework to study farmers' decisions on adoption of innovation: insights from expected utility theory and theory of planned behavior. African Journal of Agricultural Research, 10(29), 2814-2825.

Cameron, A. C., \& Trivedi, P. K. (2005). Microeconometrics: methods and applications. New York: Cambrigde University Press.

Centro de Estudos Avançados em Economia Aplicada CEPEA. (2015). Produto Interno Bruto (PIB) do agronegócio. Recuperado em: 08 de outubro de 2017, de http: / / www. cepea.esalq.usp.br

Climate Research Unit - CRU. (2013). CRU TS Data Base v. 3.21. University of East Anglia. Recuperado em: 08 de outubro de 2017, de https:/ / crudata.uea.ac.uk/cru/ data/hrg/

Cunha, D. A., Coelho, A. B., \& Féres, J. G. (2015). Irrigation as an adaptive strategy to climate change: an economic perspective on Brazilian agriculture. Environment and Development Economics, 20, 57-79.

Debela, N., Mohammed, C., Bridle, K., Corkrey, R., \& Mcneil, D. (2015). Perception of climate change and its impact by smallholders in pastoral/agropastoral systems of Borana, South Ethiopia. Springer Plus, 4(236), 1-12.

Deressa, T. T., Hassan, R. M., \& Ringler, C. (2011). Perception of and adaptation to climate change by farmers in the Nile basin of Ethiopia. The Journal of Agricultural Science, (149), 23-31.

Edward-Jones, G. (2006). Modelling farmer decisionmaking: concepts, progress and challenges. Animal Science, 82, 783-790.

Etwire, P. M., Al-Hassan, R. M., Kuwornu, J. K. M., \& Osei-Owusu, Y. (2013). Smallholder farmers' adoption of technologies for adaptation to climate change in Northern Ghana. Journal of Agricultural Extension and Rural Development, 5, 121-129.

Filmer, D., \& Pritchett, L. H. (2001). Estimating wealth effects without expenditure data - or tears: an application to educational enrolments in States of India. Demography, 38(1), 115-1131.

Fuentes, M. C., Bastos, S. B., \& Santos, N. M. (2015). Estudo do conhecimento climático popular na região semiárida do estado da Bahia. Revista de Ciências Humanas, 15(2), 349-365.

Galdies, C., Said, A., Camilleri, L., \& Caruana, M. (2016). Climate change trends In Malta and related beliefs, concerns and attitudes toward adaptation among Gozitan farmers. European Journal of Agronomy, 74, 18-28.

Gebrehiwot, T., \& Venn, A. V. D. (2013). Farm level adaptation to climate change: the case of farmer's in the ethiopian highlands. Environmental Management, 52, 29-44.

Haden, V. R., Niles, M. T., Lubell, M., Perlman, J., \& Jackson, L. E. (2012). Global and local concerns: what attitudes and beliefs motivate farmers to mitigate and adapt to climate change? PLoS One, 7(12), e52882. 
Hagen, B. (2016). Public perception of climate change: policy and communication. London: Routledge Studies in Environmental Communication and Media.

Hansson, H., Ferguson, R., \& Olofsson, C. (2012). Psychological constructs underlying farmers' decisions to diversify or specialise their businesses - an application of theory of planned behavior. Journal of Agricultural Economics, 63(2), 465-482.

Hartter, J. (2009). Attitudes of rural communities toward wetlands and forest fragments around kibale national park, Uganda. Human Dimensions of Wildlife: An International Journal, 14, 433-447.

Instituto Brasileiro de Geografia e Estatística - IBGE. (2006). Censo agropecuário 2006: Brasil, grandes regiões e unidades da federação. Rio de Janeiro: IBGE.

Instituto Brasileiro de Geografia e Estatística - IBGE. (2010). Censo 2010: Brasil, grandes regiões e unidades da federação. Rio de Janeiro: IBGE.

Intergovernmental Panel on Climate Change-IPCC. (2014). Part a: global and sectoral aspects. In C. B. Field, \& V. R. Barros (Eds.), Climate change 2014: impacts, adaptation, and vulnerability. Cambridge: Cambridge University Press.

Kpadonou, R. A. B., Adégbola, P. Y., \& Tovignan, S. D. (2012). Local Knowledge And adaptation to climate change in Ouémé Valley, Benin. African Crop Journal, 20, 181-192.

Lebel, L. (2013). Local knowledge and adaptation to climate change in natural resource-based societies of the Asia-Pacific. Mitigation and Adaptation Strategies for Global Change, 18(7), 1057-1076.

Lemos, M. C., \& Kirchhoff, C. J. (2016). Climate information and water management: building adaptive capacity or business as usual? In K. Conca \& E. Weinthal (Eds.), The oxford handbook of water politics and policy. Oxford: Oxford University Press.

Lemos, M. C., Finan, T. J., Fox, R. W., Nelson, D. R., \& Tucker, J. (2002). The use of seasonal climate forecasting in policymaking: lessons from northeast Brazil. Climatic Change, 55, 479-507.

Li, S., Juhász-Horváth, L., Harrison, P. A., Pintér, L., \& Rounsevell, M. D. A. (2017). Relating farmer's perceptions of climate change risk to adaptation behavior in Hungary. Journal of Environmental Management, 185, 21-30.

Mabe, F. N., Sienso, G., \& Donkoh, S. (2014). Determinants of choice of climate change adaptation strategies in Northern Ghana. Research in Applied Economics, 6(4), 75-94.

Machado-Filho, H. O. (2015). Climate change and its impacts on family farming in the north/northeast regions of Brazil. Brasília: International Policy Centre for Inclusive Growth, United Nations Development Programme.

Maksoud, H. (1964). Hidrologia e possibilidades hidroenergéticas da bacia do Rio das Contas, na Bahia. Rio de Janeiro: IBGE.

Mandleni, B., \& Anim, F. D. K. (2011). Climate change awareness and decision on adaptation measures by livestock farmers in South Africa. The Journal of Agricultural Science, 3(3), 258-268.
Marengo, J. A., Alves, L. M., Alvala, R. C. S., Cunha, A. P., Brito, S., \& Moraes, O. L. L. (2017). Climatic characteristics of the 2010-2016 drought in the semiarid Northeast Brazil region. Anais da Academia Brasileira de Ciências (in press).

Menike, L. M. C. S., \& Arachchi, K. A. G. P. K. (2016). Adaptation to climate change by smallholder farmers in rural communities: evidence from Sri Lanka. Procedia Food Science, 6, 288-292.

Moon, H. R., \& Perron, B. (2008). Seemingly unrelated regressions. In S. N. Durlauf \& L. E. Blume (Eds.), The new palgrave dictionary of economics. Basingstoke: Palgrave Macmillan.

Moore, F. C., \& Lobell, D. B. (2014). Adaptation potential of European agriculture in response to climate change. Nature Climate Change, 4, 610-614.

Mudzonga, E. (2012). Farmers' adaptation to climate change in chivi district of Zimbabwe. Harare: Trade and Development Studies Centre.

Naess, L. O. (2013). The role of local knowledge in adaptation to climate change. Wiley Interdisciplinary Reviews: Climate Change, 4, 99-106.

Nelson, G. C., Valin, H., Sands, R. D., Havlík, P., Ahammad, H., Deryng, D., Elliott, J., Fujimori, S., Hasegawa, T., Heyhoe, E., Kyle, P., Lampe, M. V., Lotze-Campen, H., D'Croz, D. M., Meijl, H. V., Mensbrugghe, D. V. D., Müller, C., Popp, A., Robertson, R., Robinson, S., Schmid, E., Scmitz, C., Tabeau, A., \& Willenbockel, D. (2014). Climate change effects on agriculture: economic responses to biophysical shocks. Proceedings of the National Academy of Sciences, 111(9), 3274-3279.

Nguyen, T. P. L., Seddaiu, G., Virdis, S. G. P., Tidore, C., Pasqui, M., \& Roggero, P. P. (2016). Perceiving to learn or learning to perceive? Understanding farmers' perceptions and adaptation to climate uncertainties. Agricultural Systems, 143, 205-216.

Niles, M. T., \& Mueller, N. D. (2016). Farmer perceptions of climate change: Associations with observed temperature and precipitation trends, irrigation, and climate beliefs. Global Environmental Change, 39, 133-142.

Nunes, C. R. P. (2016). As mudanças climáticas a partir da implantação de empresas de capital estrangeiro no Nordeste: estado regulador? In A. R. Araújo, G. P. N. Belchior \& T. E. Viegas (Orgs.), Os impactos das mudanças climáticas no Nordeste brasileiro (pp. 17-34). Fortaleza: Fundação Sintaf.

Obermaier, M., \& Rosa, L. P. (2013). Mudança climática e adaptação no Brasil: uma análise crítica. Estudos Avançados, 27(78), 155-176.

Paula, F. C. F., Lacerda, L. D., Marins, R. V., Aguiar, J. E., Ovalle, A. R. C., \& Falcão Filho, C. A. T. (2010). Emissões naturais e antrópicas de metais e nutrientes para a bacia inferior do Rio das Contas, Bahia. Quimica Nova, 33(1), 70-75.

Peel, M. C., Finlayson, B. L., \& Mcmahon, T. A. (2007). Updated world map of the Köppen-Geiger climate classification. Hydrology and Earth System Sciences, 11, 1633-1644. 
Pires, M. V., \& Cunha, D. A. (2014). Climate change and adaptive strategies in Brazil: the economic effects of genetic breeding. Revista de Economia e Sociologia Rural, 52(4), 627-642.

Pires, M. V., Cunha, D. A., Reis, D. I., \& Coelho, A. B. (2014). Percepção de produtores rurais em relação às mudanças climáticas e estratégias de adaptação no estado de Minas Gerais, Brasil. Revista de Ciências Agrárias (Belém), 37(4), 431-440.

Rosenzweig, C., Elliot, J., Deryng, D., Ruane, A. C., Müller, C., Arneth, A., Boote, K. J., Folberth, C., Glotter, M., Khabarov, N., Neumann, K., Piontek, F., Pugh, T. A. M., Schmid, E., Stehfest, E., Yang, H., \& Jones, J. W. (2014). Assessing agricultural risks of climate change in the 21st century in a global gridded crop model intercomparison. Proceedings of the National Academy of Sciences, 111(9), 3268-3273.

Schembergue, A., Cunha, D. A., Carlos, S. M., Pires, M. V., \& Faria, R. M. (2017). Sistemas agroflorestais como estratégia de adaptação aos desafios das mudanças climáticas no Brasil. Revista de Economia e Sociologia Rural, 55(1), 9-30.

Superintendência de Estudos Econômicos e Sociais da Bahia - SEI. (1998). Atributos climáticos do estado da bahia. Salvador: SEI.
Temesgen, D., Yehualashet, H., \& Rajan, D. S. (2014). Climate change adaptations of smallholder farmers in South Eastern Ethiopia. Journal of Agricultural Extension and Rural Development, 6(11), 354-366.

Tesfahunegn, G. B., Mekonen, K., \& Tekle, A. (2016). Farmers' perception on causes, indicators and determinants of climate change in northern Ethiopia: Implication for developing adaptation. Applied Geography, 73, 1-12.

Uddin, M. N., Bokelmann, W., \& Entsminger, J. S. (2014). Factors affecting farmers' adaptation strategies to environmental degradation and climate change effects: a farm level study in Bangladesh. Climate, 2, 223-241.

Wamsler, C., Brink, E., \& Rentala, O. (2012). Climate change, adaptation, and formal education: the role of schooling for increasing societies' adaptive capacities in el Salvador and Brazil. Ecology and Society, 17(2)

Wauters, E., Bielders, C., Poesen, J., Govers, G., \& Mathijs, E. (2010). Adoption of soil conservation practices in Belgium: an examination of the theory of planned behavior in the agri-environmental domain. Land Use Policy, 27, 86-94.

Zeleke, M. T., \& Aberra, Y. (2014). Determinants of the adoption of land management strategies against climate change in Northwest Ethiopia. Ethiopian Renaissance Journal of Social Sciences and the Humanities, 1(1), 51-64. 
Anexo. Índice de percepção climática

O Índice de Percepção Climática foi construído tendo como base a proposição metodológica de Filmer \& Pritchett (2001):

$I=\sum_{j}\left[f_{k} \frac{\left(a_{j}-\bar{a}_{j}\right)}{s_{j}}\right]$

em I representa o valor do índice para cada agricultor da amostra; cada variável que compõe o índice é identificada por $j$, assim como cada produtor é indexado por $i$. O valor observado da variável $j$ para o produtor $i$ é dado por $a j_{i}$; $\bar{a}_{j}$ indica o valor médio da variável $j$ e $s_{j}$ representa o desvio padrão da variável $j$. O valor $f_{j}$ é o peso dado a cada variável na composição do índice.

De acordo com a literatura, há três formas de se determinar o peso dado a cada variável a ser utilizada nos índices: (i) julgamento prévio do pesquisador; (ii) pesos iguais às variáveis; (iii) métodos estatísticos. No presente estudo, que segue a proposição metodológica de Filmer \& Pritchett (2001), considerou-se que as variáveis que compõem o índice não afetam igualmente a percepção climática. Dessa forma, foi considerado o método estatístico de Análise Fatorial por Componentes Principais para obter a ponderação das variáveis.

A descrição e as estatísticas descritivas das variáveis utilizadas na construção do Índice de Percepção Climática são apresentadas na Tabela A1.

Tabela A1. Descrição e estatísticas descritivas das variáveis utilizadas na construção do Índice de Percepção Climática

\begin{tabular}{|c|c|c|c|}
\hline Questões & Opções de respostas & Média & Desvio padrão \\
\hline $\begin{array}{l}\text { 1. Você já observou/percebeu alguma condição } \\
\text { climática extrema? }\end{array}$ & $\begin{array}{l}\text { Não sei responder }=0 \\
\text { Não, de modo nenhum = } 1 \\
\text { Não, talvez não }=2 \\
\text { Sim, talvez }=3 \\
\text { Sim, com certeza }=4\end{array}$ & 3,71 & 0,82 \\
\hline 2. Em sua opinião, o último inverno foi: & $\begin{array}{l}\text { Não percebi alterações }=0 \\
\text { Mais frio que o normal = } 1 \\
\text { Mais quente que o normal = } 2\end{array}$ & 1,41 & 0,75 \\
\hline $\begin{array}{l}\text { 3. Ainda em relação ao último inverno, você acha } \\
\text { que nele: }\end{array}$ & $\begin{array}{l}\text { Não percebi alterações }=0 \\
\text { Choveu mais do que o normal }=1 \\
\text { Choveu menos do que o normal }=2\end{array}$ & 1,57 & 0,70 \\
\hline 4. Em sua opinião, o último verão foi: & $\begin{array}{l}\text { Não percebi alterações = } 0 \\
\text { Mais frio do que o normal = } 1 \\
\text { Mais quente do que o normal = } 2\end{array}$ & 1,43 & 0,81 \\
\hline 5. Ainda em relação ao último verão, você acha que nele: & $\begin{array}{l}\text { Não percebi alterações }=0 \\
\text { Choveu mais do que o normal }=1 \\
\text { Choveu menos do que o normal }=2\end{array}$ & 1,51 & 0,72 \\
\hline
\end{tabular}

Fonte: Dados da pesquisa.

Após a estimação do modelo de Análise Fatorial por Componentes Principais (Tabela A2), as variáveis foram relacionadas com os fatores através das cargas fatoriais de modo a atribuir os pesos para cada variável presente no índice. Os pesos foram obtidos a partir dos fatores rotacionados pelo método varimax (Tabela A3). 
Tabela A2. Resultados da Análise Fatorial por Componentes Principais

\begin{tabular}{ccccc}
\hline Fator & Eigenvalue & Diferença & Proporção & Proporção acumulada \\
\hline 1 & 1,3285 & 0,2183 & 0,2657 & 0,2657 \\
2 & 1,1102 & 0,1934 & 0,2220 & 0,4878 \\
3 & 0,9167 & 0,0773 & 0,1834 & 0,6711 \\
4 & 0,8394 & 0,0344 & 0,1679 & 0,8390 \\
5 & 0,8049 & - & 0,1610 & 1,0000 \\
\hline
\end{tabular}

Teste LR: $\chi^{2}=25.83$; Prob $>\chi^{2}=0,0040$. Fonte: Dados da pesquisa.

Tabela A3. Pesos considerados na construção do Índice de Percepção Climática

\begin{tabular}{ll}
\hline \multicolumn{1}{c}{ Variáveis } & Peso \\
\hline Observou ou percebeu evento extremo & 0,51 \\
Percebeu alterações na temperatura de verão & 0,48 \\
Percebeu alterações na temperatura de inverno & 0,45 \\
Percebeu alterações na precipitação de verão & 0,24 \\
Percebeu alterações na precipitação de inverno & $-0,06$ \\
\hline Fonte: Dados da pesquisa. &
\end{tabular}

